\title{
iRGD-modified lipid-polymer hybrid nanoparticles loaded with isoliquiritigenin to enhance anti-breast cancer effect and tumor-targeting ability
}

This article was published in the following Dove Press journal:

International Journal of Nanomedicine

I June 2017

Number of times this article has been viewed

\author{
Fei Gaol-3 \\ Jinming Zhang ${ }^{3}$ \\ Chaomei $\mathrm{Fu}^{3}$ \\ Xiaoming $\mathrm{Xie}^{4}$ \\ Fu Peng ${ }^{1-3}$ \\ Jieshu You ${ }^{1,2}$ \\ Hailin Tang ${ }^{1,2,4}$ \\ Zhiyu Wang ${ }^{5}$ \\ Peng $\mathrm{Li}^{6}$ \\ Jianping Chen ${ }^{1-3}$
}

'School of Chinese Medicine, Li Ka Shing Faculty of Medicine, University of Hong Kong, Hong Kong, ${ }^{2}$ Shenzhen Institute of Research and Innovation, University of Hong Kong, Shenzhen,

${ }^{3}$ College of Pharmacy, Chengdu

University of Chinese Medicine,

Chengdu, ${ }^{4}$ Department of Breast

Oncology, Sun Yat-Sen University

Cancer Center, State Key Laboratory

of Oncology in South China,

Collaborative Innovation Center

for Cancer Medicine, ${ }^{5}$ Department

of Mammary Disease, Guangdong

Provincial Hospital of Chinese

Medicine, Guangzhou, ${ }^{6}$ State Key

Laboratory of Quality Research

in Chinese Medicine, Institute of

Chinese Medical Sciences, University

of Macau, Macau, People's Republic

of China

Correspondence: Jianping Chen

School of Chinese Medicine, Li Ka Shing

Faculty of Medicine, University of Hong

Kong, I 0 Sassoon Road, Pokfulam,

Hong Kong 999077

Tel +852 95I 47068

Fax +852 28725476

Email abchen@hku.hk
Abstract: Isoliquiritigenin (ISL), a natural anti-breast cancer dietary compound, has poor delivery characteristics and low bioavailability. In order to promote the therapeutic outcome of ISL, a tumor-targeting lipid-polymer hybrid nanoparticle (NP) system modified by tumorhoming iRGD peptides has been developed. The hybrid NPs were prepared by a modified single-step nanoprecipitation method to encapsulate ISL. iRGD peptides were anchored on the surface by a postinsertion method (ISL-iRGD NPs). The stable lipid-polymer structure of ISL-iRGD NPs, with high encapsulation and loading efficiency, was confirmed. Compared to free ISL and non-iRGD-modified counterparts, ISL-iRGD NPs showed higher cytotoxicity and cell apoptosis against the different type of breast cancer cells. This was attributable to higher cellular accumulation mediated by the iRGD-integrin recognition and the nanoscale effect. More importantly, based on the active tumor-tissue accumulation by iRGD peptides and the prolonged in vivo circulation by the stealth nanostructure, ISL-iRGD NPs displayed higher tumor-growth inhibition efficiency in 4T1-bearing breast-tumor mouse models. Therefore, the constructed iRGD modified lipid-polymer hybrid NPs would provide a promising drug-delivery strategy to improve ISL in anti-breast cancer efficacy.

Keywords: isoliquiritigenin, iRGD, lipid-polymer hybrid NPs, breast cancer

\section{Introduction}

The morbidity of breast cancer has ranked first in female cancers for a long time worldwide. In some developed regions, breast cancer is still the second-commonest major cause of cancer death. ${ }^{1,2}$ Currently chemotherapy remains the irreplaceable method in treating breast cancer in clinic, yet it has limited efficacy and can produce some serious side effects for patients. ${ }^{3-6}$ On the other hand, seeking natural compounds in treating breast cancer has become a promising research area and attracted a lot of scholars, due to some compounds' high efficacy and minor side effects, such as curcumin, (-)-epigallocatechin gallate, and emodin. ${ }^{7}$ In very recent years, we have found a flavonoid, isoliquiritigenin (ISL; structure in Figure 1A), that is not only able to inhibit angiogenesis of breast cancer cells via the VEGF-VEGFR2 pathway ${ }^{8}$ and prevent mammary carcinogenesis through WIF1 demethylation, ${ }^{9}$ but can also chemosensitize breast cancer stem cells, ${ }^{10}$ serving as an autophagy inducer regulating chemoresistance-associated autophagy. ${ }^{11}$ In addition, studies have shown that ISL can treat breast cancer via NFKB signaling and the PI3K-Akt pathway, inhibiting p38 expression and downregulating AA-metabolizing enzymes. ${ }^{12-14}$ This evidence has demonstrated that ISL can be a promising anti-breast cancer agent worthy of being 


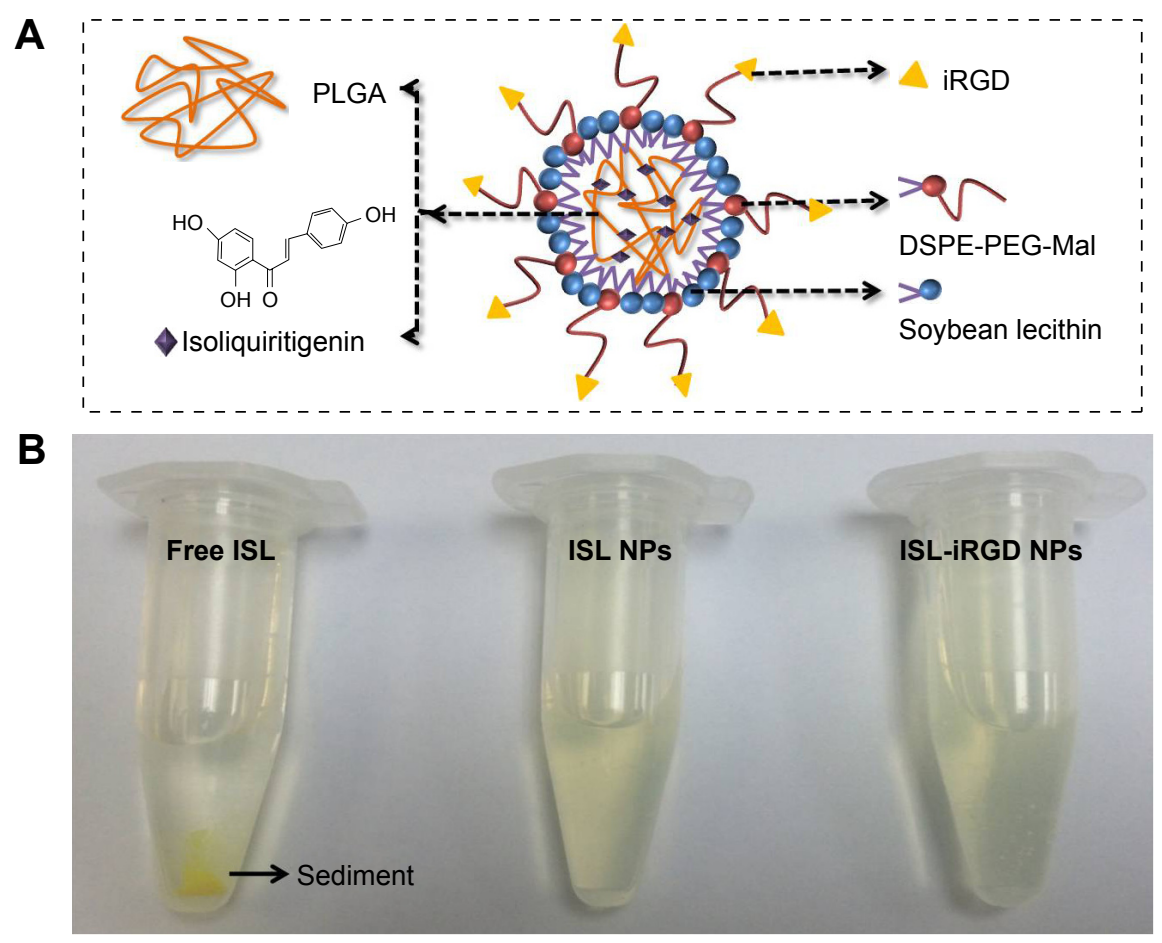

Figure I Image and structure of ISL-iRGD NPs and ISL.

Notes: Schematic representation of ISL-iRGD NPs (A); photographic image of different drugs (free ISL, ISL NPs and ISL-iRGD NPs) in aqueous solution (B).

Abbreviations: ISL, isoliquiritigenin; NPs, nanoparticles; PLGA, poly(lactic-co-glycolic acid); DSPE, I,2-distearoyl-sn-glycero-3-phosphoethanolamine; PEG, polyethylene glycol; Mal, maleimide.

developed. However, currently its use in clinical application is limited by several factors, such as poor solubility, low bioavailability, high effective dose $(50 \mathrm{mg} / \mathrm{kg}$ mouse body weight), and poor targeting ability (very low concentrations in mammary tissue). ${ }^{9}, 15$ Therefore, dealing with these problems is a key process in its development.

Nanoparticles (NPs) are a typical nanodelivery system that can improve solubility, enhance bioavailability, and increase targeting distribution of a drug ${ }^{16}$ while lipidpolymer NPs represent a promising delivery platform that combines the biomimetic advantages of liposomes and mechanical advantages of biodegradable polymeric NPs. ${ }^{17,18}$ In addition, modification of targeting peptides on the surface of the NPs serves as a smart design for enhancing targeting distribution. ${ }^{19}$ iRGD peptides, nine-unit cyclic (CRGDK/ RGPD/EC) tumor-homing peptides with an RGD sequence were selected, since they bind preferentially to integrin $\alpha_{\mathrm{v}} \beta_{3}$ receptors, $\alpha_{v} \beta_{5}$ integrin receptors and Nrp1 receptors. Firstly, the iRGD peptide can directly bind to $\alpha \beta$ integrin, which is overexpressed on the surface of cancer cells and tumor-vessel cells but not in normal vessel cells, and then proteolytically turns into CRGDK/R, exposing an active CendR motif at the C-terminus. Finally, the CendR motif binds with the permeability-regulating receptor $\mathrm{Nrp} 1$ in cancer cells. ${ }^{20-23}$
Therefore, modified iRGD peptides on the surface of lipid-polymer NPs represent an effective design.

To the best of our knowledge, recent attempts to improve ISL delivery via nanosystems against cancer have been very limited and the outcomes not very satisfactory, ie, in the form of nanomaterial toxicity and poor targeting ability of existent NPs. ${ }^{24,25}$ Herein, iRGD-modified lipid-polymer NPs were used to encapsulate ISL (structure of NPs in Figure 1A). The basic characteristics, pharmacological efficacy, and targeting ability of the novel NPs were studied and are evaluated in this paper.

\section{Materials and methods Materials and reagents}

The animal experiments were approved by the ethics committee of the University of Hong Kong (HKU, permit 3684-15), and we followed the animal-welfare guidelines and basic regulations of Laboratory Animal Unit in HKU. ISL (purity $>97 \%$ ) was bought from Thermo Fisher Scientific (Waltham, MA, US). 1,2-Distearoyl-sn-glycero3-phosphoethanolamine- $N$-(maleimide[polyethylene glycol $_{2,000}$ ) (DSPE-PEG ${ }_{2,000}$-Mal) was purchased from Laysan Bio (Arab, AL, US). Poly(lactic-co-glycolic acid) (PLGA)-COOH (intrinsic viscosity [dL/g] 0.08, molecular 
weight 5,000 Da) was obtained from Dalian Biotechnology (Nanjing, China). Lecithin was bought from Solarbio Science and Technology (Beijing, China). Coumarin 6 ( $\mathrm{C}_{6}$; laser grade) was obtained from Aladdin Reagent (Shanghai, China). 3-(4,5-Dimethylthiazol-2-yl)-2,5-diphenyltetrazolium bromide (MTT) and Hoechst 33342 were purchased from Thermo Fisher Scientific. A fluorescein isothiocyanate (FITC)-annexin V apoptosis-detection kit used to investigate apoptosis activity was obtained from BD Biosciences (San Jose, CA, US). All reagent water was pretreated with the Milli-Q Plus system (EMD Millipore, Billerica, MA, US). All solvents, including dimethyl sulfoxide (DMSO), ethanol, acetonitrile, and methanol, were of chromatographic grade and used without further treatment.

\section{Preparation of ISL-iRGD NPs}

ISL-iRGD lipid-polymer NPs composed of a polymeric PLGA core coated with a layer of lipids and a PEG layer were prepared by modified single-step nanoprecipitation, based on a previous study. ${ }^{26}$ In brief, DSPE-PEG-Mal (6 $\mathrm{mg}$ ) and lecithin (4 mg) solution were dissolved in $0.5 \mathrm{~mL}$ alcohol and then added to $9 \mathrm{~mL}$ water. The DSPE-PEGlecithin solution was stirred and heated to $65^{\circ} \mathrm{C}$, to ensure all lipid was in liquid phase. Additionally, $1.2 \mathrm{mg}$ of ISL and $10 \mathrm{mg}$ of PLGA was dissolved in $0.5 \mathrm{~mL}$ of alcohol and acetonitrile, respectively. The PLGA solution mixed with ISL was then added to a prepared liquid-lipid solution dropwise under stirring at $700 \mathrm{rpm}$ for 6 hours at room temperature. The remaining organic solvent was evaporated in a vacuum, and the nanosuspension was filtered with a $0.45 \mu \mathrm{m}$ filter to remove free molecules. Finally, iRGD peptides were modified on the surface of NPs by a postinsertion approach based on the mild conjugation between the Mal group on the PEG layer and the terminal sulfhydryl group of iRGD. iRGD (1 mg) was added to the ISL-loaded NP suspension for 30 minutes' shaking at room temperature.

\section{Physical property characterization}

The objective of studying property characterization was to evaluate the general physical properties and basic structures of the NPs, as well as to discover evidence to confirm whether the drugs were loaded into the NPs or not. NPs were characterized by particle size, $\zeta$-potential, X-ray diffraction, infrared radiation, differential scanning calorimetry (DSC), and transmission electron microscopy (TEM). Particle size and $\zeta$-potential were both detected by dynamic light scattering (Malvern Instruments, Malvern, UK) at $25^{\circ} \mathrm{C}$. The morphology of NPs was detected by TEM (Tecnai G20; Thermo
Fisher Scientific) at operation voltage of $200 \mathrm{kV}$. Fouriertransform infrared spectra (Nicolet iS 50; Thermo Fisher Scientific) were employed to characterize the encapsulation of ISL in NPs. Thermodynamic properties of ISL-iRGD NPs were detected by diamond DSC (PerkinElmer, Waltham, MA, US). The crystallographic structures of NPs were characterized by X-ray diffraction (Bruker Optik GmbH, Ettlingen, Germany).

\section{$\mathrm{EE}, \mathrm{LE}$, and stability analysis}

Loading efficiency (LE) and encapsulation efficiency (EE) of ISL were determined with high-performance liquidchromatography (HPLC) (Dionex Ultimate 3000; Thermo Fisher Scientific) equipped with a $\mathrm{C}_{18}$ column $(250 \times 4.6 \mathrm{~mm})$. The mobile phase was methanol/water $(70 / 30, \mathrm{v} / \mathrm{v})$ with a detection wavelength of $306 \mathrm{~nm}$. The flow rate was $1 \mathrm{~mL} / \mathrm{min}$. Methanol was used to disrupt the structure of NPs and dissolved ISL before it was analyzed by HPLC. Calculations of EE and LE were as follows:

$$
\begin{gathered}
\text { EE }(\%)=\frac{\text { Amount of ISL loaded }}{\text { Amount of ISL added }} \times 100 \% \\
\text { LE }(\%)=\frac{\text { Amount of ISL loaded }}{\text { Amount of ISL added }+ \text { polymer }} \times 100 \%
\end{gathered}
$$

In addition, change in particle size, $\zeta$-potential, EE, and $\mathrm{LE}$ for 15 days during storage at $25^{\circ} \mathrm{C}$ was carried out as the stability evaluation.

\section{Cell culture}

Human breast cancer cell lines (MDA-MB231 and MCF7) and a mouse breast cancer cell line (4T1) were obtained from the American Type Culture Collection (Manassas, VA, US). Cells were cultured in medium (Dulbecco's Modified Eagle's Medium for MDA-MB231 and 4T1, Roswell Park Memorial Institute 1640 for MCF7) supplemented with 10\% fetal bovine serum (Thermo Fisher Scientific) and 1\% streptomycin and penicillin (Thermo Fisher Scientific). For all the in vitro experiments, free ISL was dissolved in DMSO in advance, and then diluted over 1,000-fold. All of the NPs or drugs were diluted by uncompleted medium (supplemented with $0.5 \%$ fetal bovine serum and 1\% streptomycin and penicillin).

\section{Cell-proliferation assays}

MTT assay was used to evaluate the cell proliferation activity of different breast cancer cells. In brief, MDA-MB231, 4T1, and MCF7 cells were seeded onto the 96-well plates at a 
density of $4 \times 10^{3}$ cells per well. Four groups (ISL-iRGD NPs, ISL NPs, free ISL, and blank NPs) were added to the wells with six duplicates for each concentration. After incubation for 24 hours, $20 \mu \mathrm{L}$ MTT solution $(5 \mathrm{mg} / \mathrm{mL})$ was added into each well for 4 hours' incubation. The MTT-added solution was then removed following the addition of $100 \mu \mathrm{L}$ of DMSO to each well and mixed thoroughly with pipette. Afterward, the samples were incubated at $37^{\circ} \mathrm{C}$ for 10 minutes and mixed. Absorbance was read at $540 \mathrm{~nm}$.

\section{Apoptosis assays}

The FITC-annexin V apoptosis-detection kit was used to determine the effects of cancer-cell apoptosis. MDAMB231 breast cancer cells were treated with the four drug groups (ISL-iRGD NPs, ISL NPs, free ISL, and blank NPs) with ISL concentration of $50 \mu \mathrm{M}$ for 16 hours. Both nonadherent and adherent cells were collected. Pretreatment procedures were carried out as per the instructions of the FITC-annexin V apoptosis-detection kit. Cells were analyzed with an LSR Fortessa analyzer (BD Biosciences) within 1 hour. Annexin $\mathrm{V}-\mathrm{FITC}^{+} / \mathrm{PI}^{-}$cells were considered early apoptotic cells, and annexin $\mathrm{V}-\mathrm{FITC}{ }^{+} / \mathrm{PI}^{+}$cells were considered late apoptotic cells.

\section{Cell-uptake analysis}

Cellular uptake is an important index to estimate drug capacity to enter cells and drug concentration in cells. ${ }^{27} \mathrm{C}_{6}$, a hydrophobic fluorescent dye, was used to label the NPs, and used to investigate the cell-uptake ability of different NPs qualitatively and quantitatively. ${ }^{28}$ The preparation method for encapsulating $\mathrm{C}_{6}$ was similar to ISL NPs.

Flow cytometry (FCM) was used for quantitative studies of the cellular uptake of NPs. ${ }^{29,30}$ MDA-MB231 breast cancer cells $\left(8 \times 10^{5}\right)$ were seeded onto the six-well plates. After 12 hours' incubation, the medium was removed and the different drug solutions added at certain concentrations of $\mathrm{C}_{6}$. For the quantitative study, cells were incubated with $\mathrm{C}_{6}$-iRGD NPs, $\mathrm{C}_{6} \mathrm{NPs}$, and free $\mathrm{C}_{6}$ with an equivalent concentration of $\mathrm{C}_{6}(25 \mathrm{ng} / \mathrm{mL})$ for 4 hours at $37^{\circ} \mathrm{C}$. For dosedependent study, different concentrations of $\mathrm{C}_{6}(25,12.5$, $6.25,3.125 \mathrm{ng} / \mathrm{mL}$ ) were used for incubation with cells for 4 hours at $37^{\circ} \mathrm{C}$. Furthermore, cells were incubated with $\mathrm{C}_{6}$-iRGD NPs $(25 \mathrm{ng} / \mathrm{mL})$ at different time points $(0.5,1,2$, and 4 hours) for time-dependent study. For the energydependent study, cells were incubated with $\mathrm{C}_{6}$-iRGD NPs (25 ng/mL) at different time points $(0.5,1,2$, and 4 hours) at $37^{\circ} \mathrm{C}$ and $4^{\circ} \mathrm{C}$, respectively. Untreated cells were used as controls.
Cellular uptake of various ISL samples was observed by fluorescence microscopy. MDA-MB231 breast cancer cells $\left(5 \times 10^{4}\right)$ were seeded onto the six-well plates. After 24 hours' incubation, the completed medium was removed and different drug solutions at a concentration of $\mathrm{C}_{6}(85 \mathrm{ng} / \mathrm{mL})$ added. The control group was untreated cells. After 4 hours' incubation, the drug solutions were removed and washed twice with cold phosphate-buffered saline (PBS), fixed with 4\% paraformaldehyde in PBS for 10 minutes, washed twice more with PBS, and then stained with Hoechst 33342. Intracellular Hoechst 33342 and $\mathrm{C}_{6}$ were excited and observed by fluorescence microscopy.

\section{In vivo antitumor efficacy}

Primarily, 4-week-old female nude mice were used to set up the breast-tumor mouse models. $4 \mathrm{~T} 1$ cells $\left(4 \times 10^{5}\right.$ per mouse) were injected into the mammary gland via subcutaneous inoculation. After the tumor volume had reached about $100 \mathrm{~mm}^{3}$, mice were assigned to four groups randomly: ISL-iRGD NPs, ISL NPs, free ISL ( $25 \mathrm{mg} / \mathrm{kg}$ mouse body weight), and blank NPs. Formulations were administered to mice once every 2 days by intraperitoneal injection for 20 days after disinfection. Mice in the control group were administered normal saline only. Tumor volumes and body weight of mice were recorded once every 2 days. The tumor volumes were calculated using the formula $\mathrm{V}=\mathrm{W}^{2} \times \mathrm{L} / 2$. At the end of the experiment, mice were used anesthetic to death and collected tumors, livers, lungs, and kidneys. Hematoxylin and eosin (H\&E) staining was used in studying the histological characteristics of the different tissues.

\section{Biodistribution analysis}

To visualize tumor accumulation of NPs in breast-tumor mouse models, Cy5, a near-infrared fluorescence probe, was used to label NPs based on the preparation method of ISL NPs. After injecting 4T1 breast cancer cells in the nude mice for 10 days, the mice were divided into Cy5-iRGD NPs, Cy5 NPs, and free Cy5 groups. Biodistribution analysis was conducted, described as follows. Firstly, mice were given Cy5 $(35 \mu \mathrm{g} / \mathrm{kg}$ ) via intravenous (IV) injection. Secondly, mice were imaged using an IVIS Spectrum (PerkinElmer) in vivo imaging system after injecting the $\mathrm{Cy} 5$ at four time points $(2,6,12$, and 24 hours). Finally, tumors, lungs, hearts, spleens, and kidneys were collected and imaged.

\section{Statistics}

All data were presented as means \pm standard deviation (SD). All experimental results were confirmed in at least 
three independent experiments under the same conditions. Statistical comparison was carried out with Student's $t$-test. $P<0.05$ was considered statistically significant.

\section{Results and discussion \\ Preparation and characterization of ISL-iRGD NPs}

Based on the aforementioned procedures, ISL-loaded hybrid NPs composed of a polymeric PLGA core coated by a layer of lipids and PEG were prepared, and iRGD peptides were modified on the surface of NPs by postinsertion, with the structure shown in Figure 1A. With its significant improvement in water-solubility, the ISL-loaded NP suspension appeared as a homogeneous light-yellow solution, instead of lots of sediment in free ISL solution (Figure 1B).

The average size distribution of ISL-iRGD NPs was $137.2 \pm 2.6 \mathrm{~nm}$ (relative SD 1.87\%) and the $\zeta$-potential $-34.21 \pm 1.23 \mathrm{mV}$ (relative SD 3.59\%) (Figure 2A and B). As is well known, NPs with small particle size less than $200 \mathrm{~nm}$ can accumulate in tumor cells via the enhanced permeability and retention (EPR) effect. Also, NPs with high absolute negative $\zeta$-potential suggests satisfactory stability, as well as avoidance of particle aggregation and protein adsorption in blood circulation. Therefore, these NPs would be expected to show good stability and drug accumulation in tumor-tissue. Morphologically, the TEM images of ISL-iRGD NPs and blank NPs showed that distribution was uniform and the shape round and smooth (Figure 2C and D), which also indicated the loading procedure of ISL had not influenced NP formation.

The infrared spectra of ISL-iRGD NPs and other formulations were detected to confirm the structure of NPs. A strong peak at $1,228.494 \mathrm{~cm}^{-1}$ was detected only in the free ISL, mixed free ISL, and blank NPs, which indicated this peak was specific for ISL (Figure 2E). However, this peak disappeared from the ISL NPs, which means ISL was not detected on the surface of NPs and was loaded in the core. Moreover, the peaks of 2,921.771 $\mathrm{cm}^{-1}, 2,854.272 \mathrm{~cm}^{-1}$, and $1,758.849 \mathrm{~cm}^{-1}$ were detected in mixed free ISL, blank NPs, and ISL NPs, respectively, but not free ISL itself, which is supplementary evidence to confirm ISL loading in the core of the NPs. In DSC or X-ray spectrum evaluation (Figure 2F and $\mathrm{G})$, the spectrum profile or specific peaks of blank NPs were similar to ISL NPs; the same conclusion as for infraredspectrum analysis. All the three spectra confirmed the same fact that ISL was encapsulated in the core of the NPs but not on the surface.

\section{Drug-loading and -stability evaluation in vitro}

The EE and LE of ISL-iRGD NPs in Milli-Q water were $90.8 \% \pm 1.5 \%$ and $4.13 \% \pm 0.4 \%$, respectively. The stability of ISL-iRGD NPs was tested for 15 days at $4^{\circ} \mathrm{C}$. Results are shown in Table 1. The particle size of ISL-iRGD NPs changed from $138.97 \pm 2.44$ (day 1) to $134.48 \pm 2.65 \mathrm{~nm}$ (day 15), but without significant difference $(P>0.5)$. In addition, the other three indices - $\zeta$-potential, EE, and LE - all showed slight changes from day 1 to day 15 . These findings indicated ISLiRGD NPs were stable during the 15 days.

\section{Cytotoxicity and apoptosis analysis of ISL-iRGD NPs in vitro}

To evaluate the anti-breast cancer effects of ISL-iRGD NPs in vitro, MTT and double-staining apoptosis assays were conducted. Three typical breast cancer cell lines - MCF7, MDAMB231, and 4T1 - were used in the MTT assay. These cell lines were incubated with four different formulations (blank NPs, free ISL, ISL NPs, and ISL-iRGD NPs) for 24 hours. For blank NPs, there was no obvious cytotoxicity against any cell lines, which was also evidence to eliminate the influence caused of the nanomaterials. For MCF7, ISL-iRGD NPs had better inhibition effects than free ISL at high concentrations $(>12.5 \mu \mathrm{M}, P<0.05)$ but they were not better than ISL NPs (Figure 3A). For MDA-MB231 and 4T1, ISL-iRGD NPs had better inhibition effects than ISL NPs and free ISL at all concentrations $(P<0.05)$, which means iRGD enhanced the efficacy of ISL NPs in cytotoxicity (Figure 3B and C). Moreover, this evidence indicates that MDA-MB231 and 4T1 were more sensitive to the ISL-iRGD NPs than MCF7.

Based on the high sensitivity of MDA-MB231 to the ISL-iRGD NPs in cytotoxicity assays, MDA-MB231 was selected to evaluate the apoptosis-inducing efficacy of different formulations, and the results are shown in Figure 3D and Figure S1. After incubation for 16 hours, the apoptosis rate of free ISL and ISL NPs was similar $(P>0.05)$, which indicated the nanoscale of ISL may not have contributed to the efficacy of inducing MDA-MB231-cell apoptosis. However, ISL-iRGD NPs presented higher rates of apoptosis (about 40\%) than the other four groups, which suggested the stronger efficacy may have been caused by the tumor-homing iRGD peptides $(P<0.05)$.

\section{Cell-uptake ability of ISL-iRGD NPs}

As previously mentioned, iRGD is a tumor-homing peptide that shows positive targeting ability for cancer cells. Moreover, the average size of the ISL-iRGD NPs was about 
A

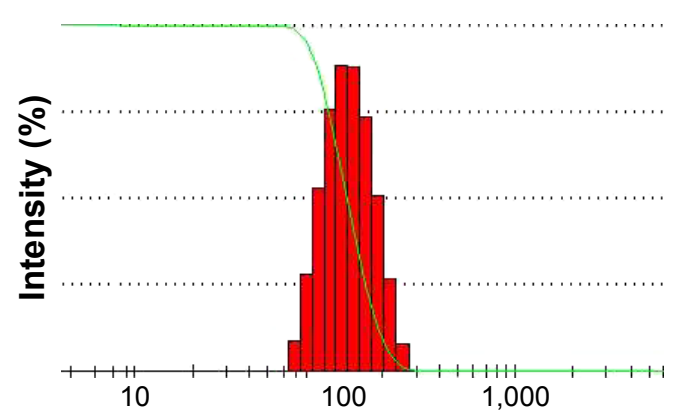

B

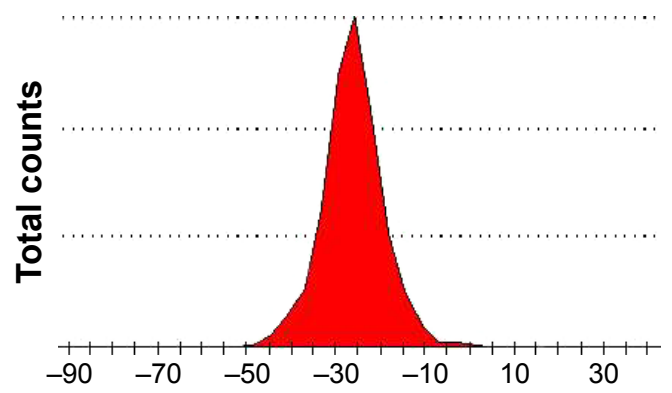

C

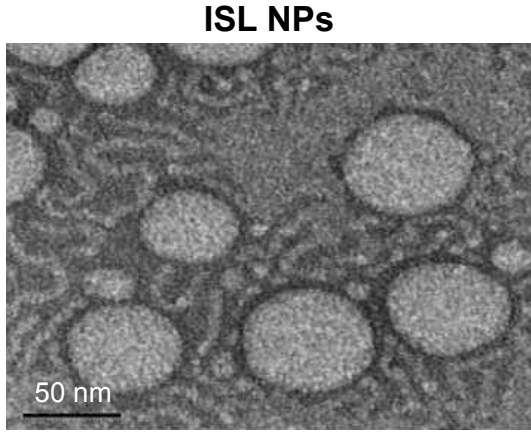

D

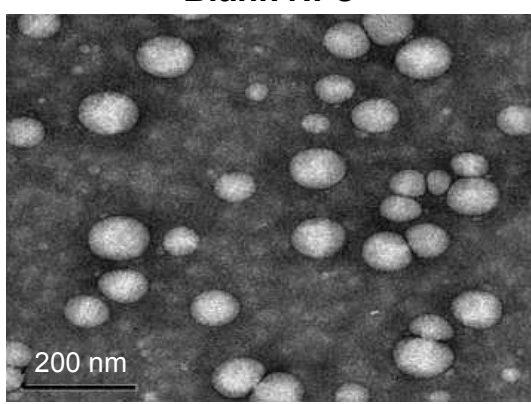

E

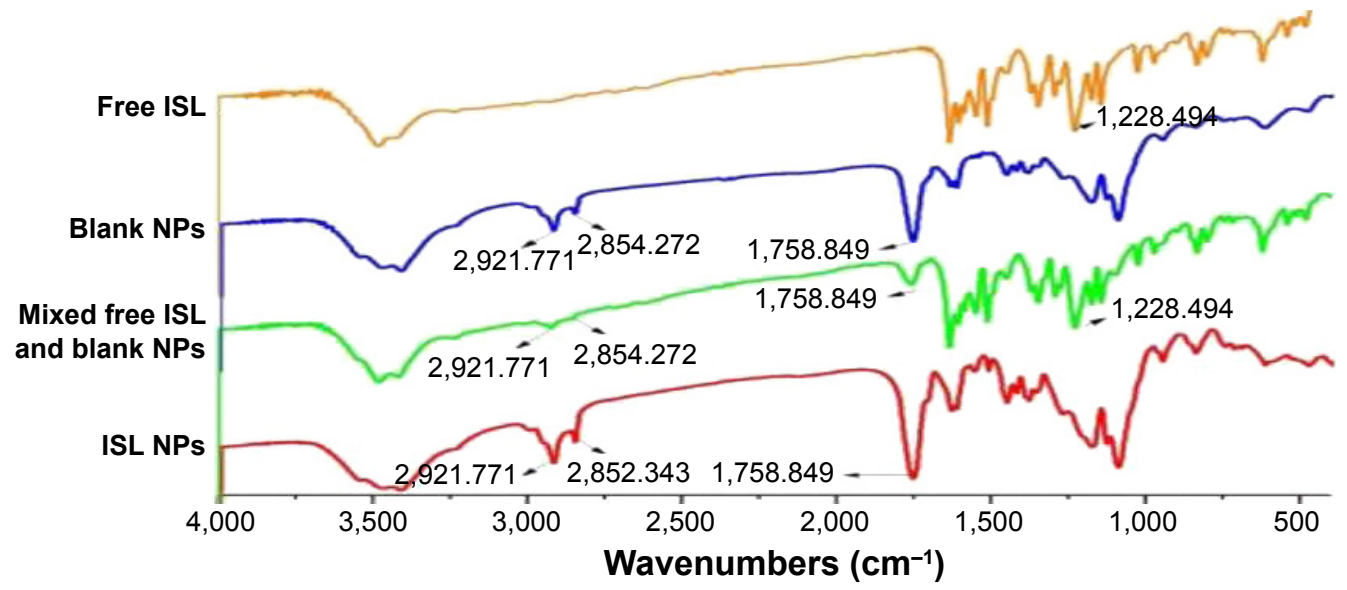

F Free ISL

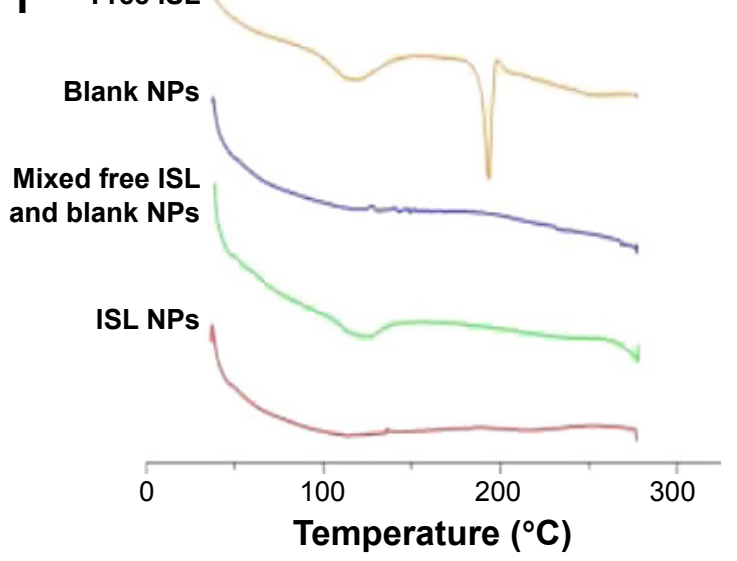

G

Free ISL

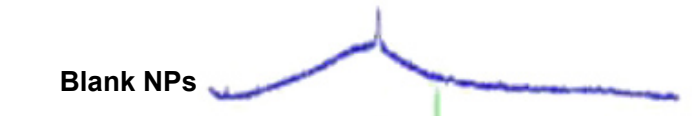

Mixed free ISL and blank NPs

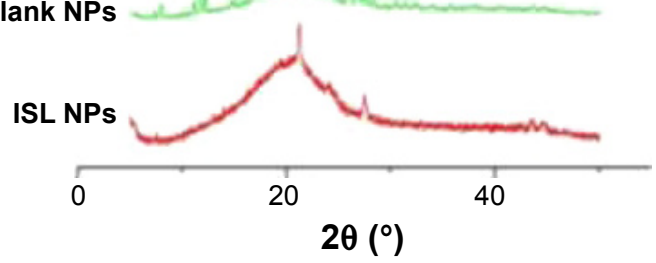

Figure 2 Characteristics of ISL-iRGD NPs.

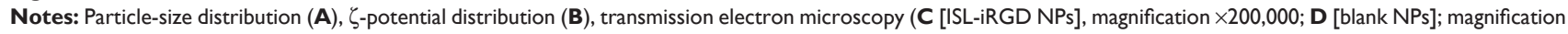
$\times 50,000$ ), infrared radiation $(\mathbf{E})$, differential scanning calorimetry $(\mathbf{F})$, and X-ray diffraction $(\mathbf{G})$ of different polymer drug-delivery systems and free drug. Abbreviations: ISL, isoliquiritigenin; NPs, nanoparticles. 
Table I Stability of ISL-iRGD lipid-polymer NPs over I5 days

\begin{tabular}{lllll}
\hline & Particle size & $\zeta$-Potential & EE & LE \\
\hline Day I & $138.97 \pm 2.44$ & $-36.15 \pm 0.74$ & $90.4 \% \pm 1.8 \%$ & $4.06 \% \pm 0.38 \%$ \\
Day I5 & $134.48 \pm 2.65$ & $-31.91 \pm 1.98$ & $87.1 \% \pm 1.2 \%$ & $3.91 \% \pm 0.4 \%$ \\
\hline
\end{tabular}

Note: All data were presented as mean \pm standard deviation.

Abbreviations: ISL, isoliquiritigenin; NPs, nanoparticles; EE, encapsulation efficiency; LE, loading efficiency.

$137.2 \mathrm{~nm}$, which is also a good size range for passive targeting of NPs. Therefore, two methods were used to evaluate the cell-uptake ability of different formulas: fluorescence microscopy and FCM.

Fluorescence microscopy is a typical method for qualitative analysis of cell-uptake ability of NPs. The intensity of the dye in the image can reflect the concentration of targetdrug uptake by certain cells indirectly. Figure 4A presents fluorescence-microscopy images of the different formulations taken up by MDA-MB231. The concentration of $\mathrm{C}_{6}$ in all formulas was $85 \mathrm{ng} / \mathrm{mL}$. After incubating with the different $\mathrm{C}_{6}$ formulations for 4 hours, $\mathrm{C}_{6}$-iRGD NPs presented the greatest intensity and were most closely situated around nuclei compared to $\mathrm{C}_{6}$ NPs and free $\mathrm{C}_{6}$, which illustrated $\mathrm{C}_{6}$-iRGD NPs were prone to internalize in MDA-MB231 cells and showed excellent targeting ability.

Qualitative analysis of the NPs was measured by FCM to confirm uptake efficacy and evaluate the manner of uptake of $\mathrm{C}_{6}$-iRGD NPs. As shown in Figure 4B, after incubation of MDA-MB231 with different $\mathrm{C}_{6}$ formulations $\left(\mathrm{C}_{6}-\mathrm{iRGD}\right.$ NPs, $\mathrm{C}_{6}$ NPs, and free $\mathrm{C}_{6}$ ) for 4 hours, the FCM-detection profiles and histogram results of mean fluorescence intensity showed the intensity of $\mathrm{C}_{6}$ in $\mathrm{C}_{6}-\mathrm{iRGD}$ NPs was strongest $(P<0.05)$, followed by $\mathrm{C}_{6}$ NPs and free ISL. This confirmed the results of fluorescence microscopy, and also illustrated the strong cell-uptake ability of ISL-iRGD NPs. Moreover, it also indicated that the high uptake capacity of the system may have been due to its nanoscale and the iRGD peptide.

The manner of uptake of $\mathrm{C}_{6}$-iRGD NPs was also detected by FCM. The FCM profile and histogram both showed the mean intensity of $\mathrm{C}_{6}$ increased with ISL dosage (Figure 4C) or time (Figure 4D and Figure S2), which indicated that the cell-uptake efficacy in MDA-MB231 of $\mathrm{C}_{6}$-iRGD NPs
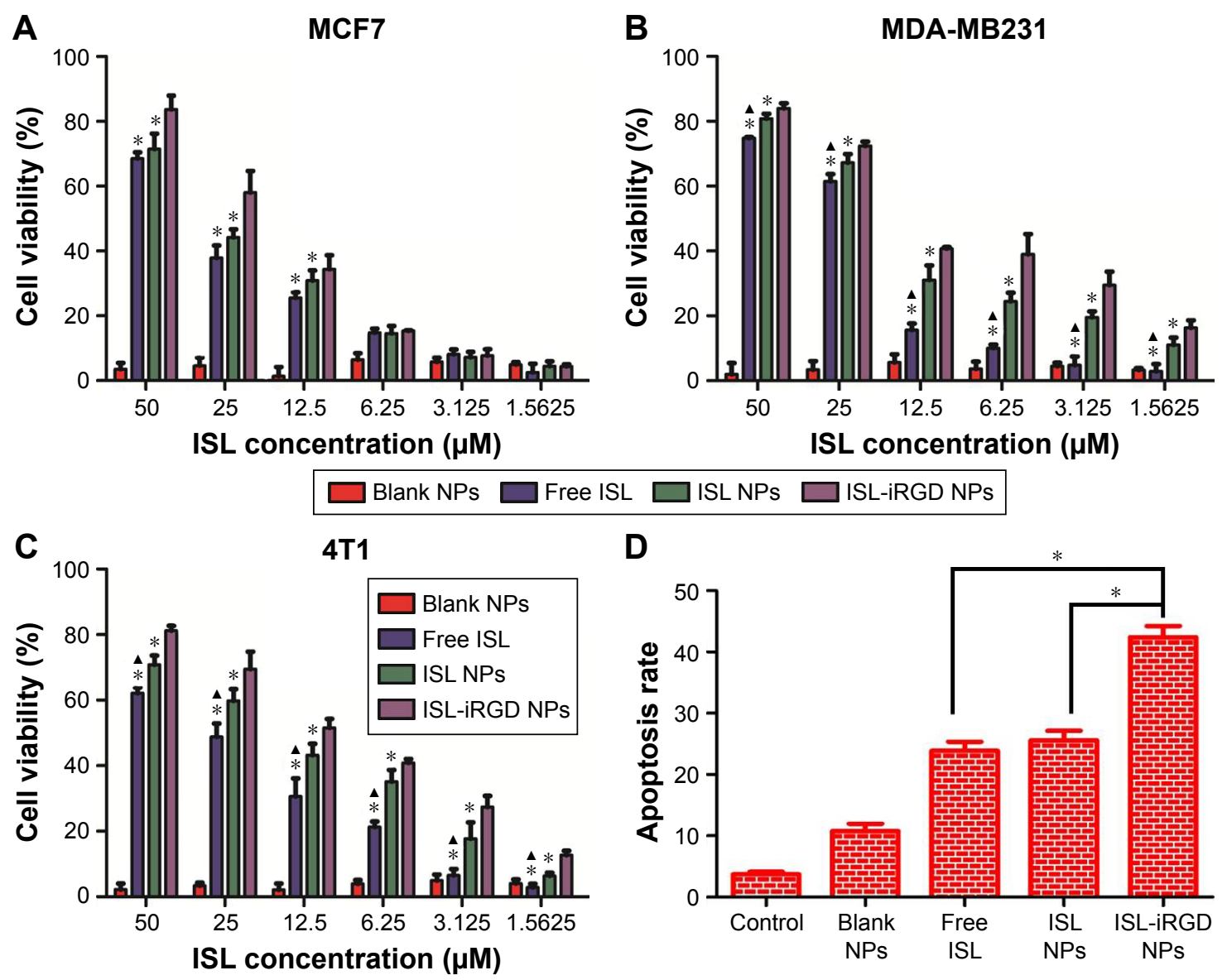

Figure 3 In vitro cytotoxicity and apoptosis analysis of ISL-iRGD NPs.

Notes: Cell viability of MCF7 (A), MDA-MB23I (B), and 4TI (C) breast cancer cell lines after incubation with ISL-iRGD NPs, ISL NPs, blank NPs and free ISL for 24 hours. Apoptosis analysis of MDA-MB23I after treated by ISL-iRGD NPs, ISL NPs, blank NPs and free ISL (D). $* P<0.05$ vs free ISL; ${ }^{\wedge}<<0.05$ vs ISL NPs.

Abbreviations: ISL, isoliquiritigenin; NPs, nanoparticles. 

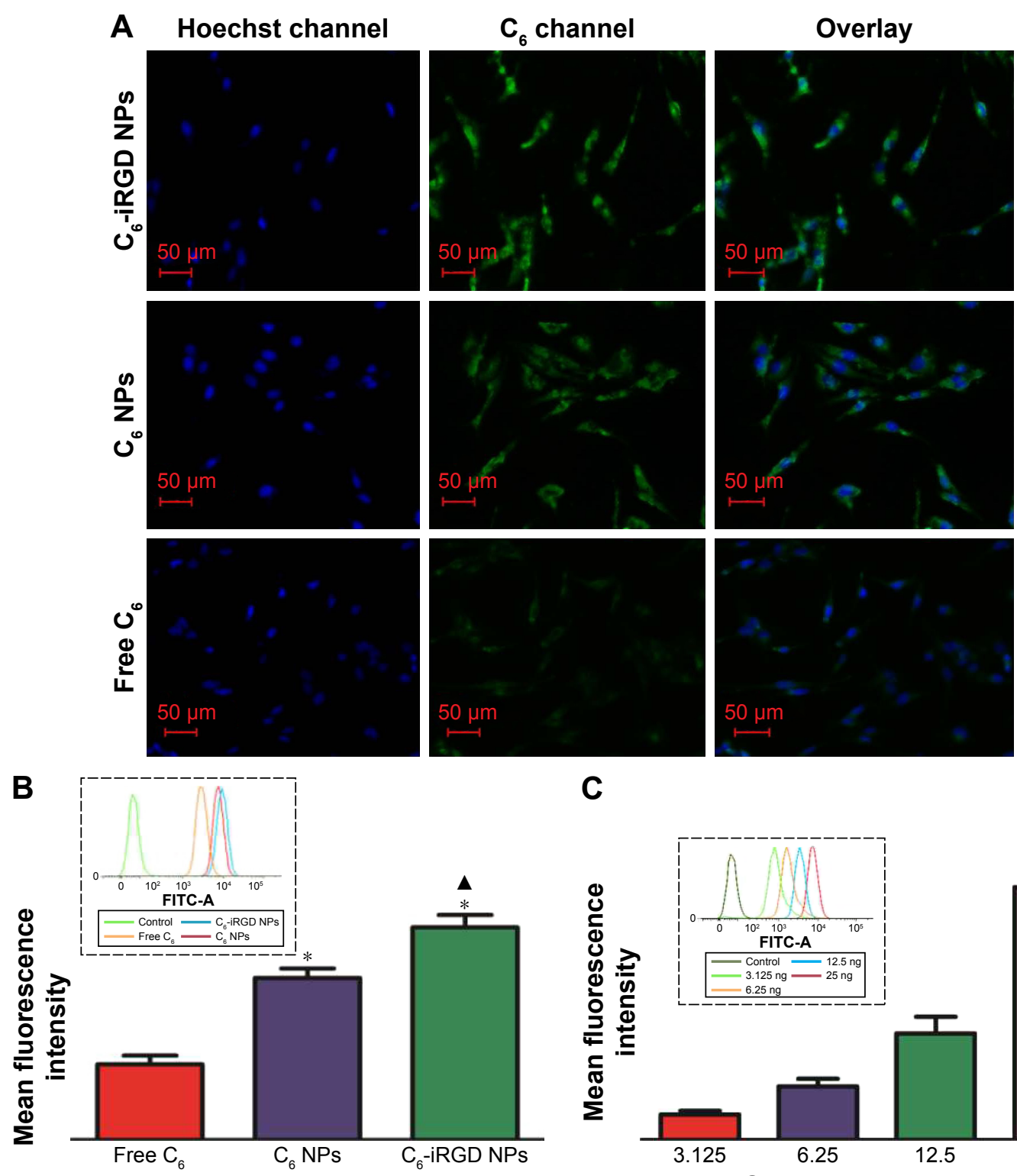

C
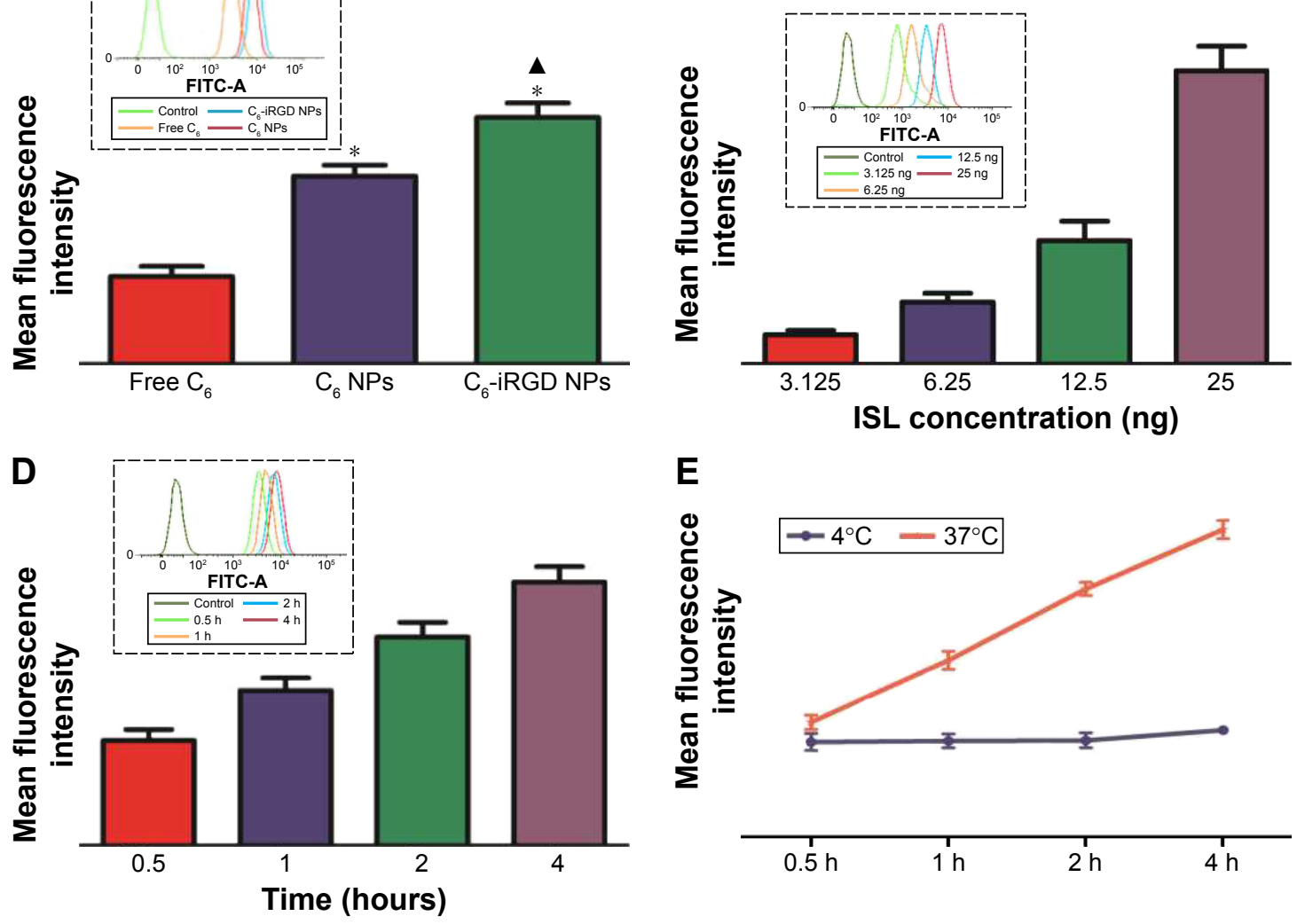

Figure 4 Cell-uptake evaluation of different $C_{6}$ formulations using the MDA-MB23I cell line.

Notes: (A) Fluorescent images of MDA-MB23I cells incubated with different $C_{6}$ formulations $\left(C_{6}\right.$-iRGD NPs, $C_{6}$ NPs, and free $C_{6}$ ) for 4 hours; magnification $\times 200$. $C_{6}$ incubation concentration was $85 \mathrm{ng} / \mathrm{mL}$. (B) Quantitative measure of $C_{6}$ uptake in different $C_{6}$ formulations $\left(C_{6}-i R G D N P s, C_{6} N P s\right.$, and free $\left.C_{6}\right)$ after incubation for 4 hours. $\mathrm{C}_{6}$-incubation concentration was $25 \mathrm{ng} / \mathrm{mL}$. $* P<0.05$ vs free $C_{6} ; \Delta P<0.05$ vs $C_{6}$ NPs. (C) Quantitative measure of $C_{6}$ uptake in $C_{6}$-iRGD NPs at different dosages $(25 \mathrm{ng} / \mathrm{mL}$, $12.5 \mathrm{ng} / \mathrm{mL}, 6.25 \mathrm{ng} / \mathrm{mL}$, and $3.125 \mathrm{ng} / \mathrm{mL}$ ) after incubation for 4 hours. (D) Quantitative measure of $\mathrm{C}_{6}$ uptake in $\mathrm{C}_{6}$-iRGD NPs at different incubation time points (0.5, I, 2 , and 4 hours). $C_{6}$ concentration was $25 \mathrm{ng} / \mathrm{mL}$. (E) Mean fluorescence intensity versus time profile after incubation with $\mathrm{C}_{6}-\mathrm{iRGD} \mathrm{NPs}$ at $4^{\circ} \mathrm{C}$ and $37^{\circ} \mathrm{C}$ for 4 hours. Abbreviations: ISL, isoliquiritigenin; NPs, nanoparticles. 
occurred in a dosage-dependent or time-dependent manner, respectively. Further analysis demonstrated that the intensity of $\mathrm{C}_{6}$ at $37^{\circ} \mathrm{C}$ is stronger than at $4^{\circ} \mathrm{C}$ at all time points (Figure 4E), and intensity increased obviously with time at $37^{\circ} \mathrm{C}$ but not at $4^{\circ} \mathrm{C}$, which suggested uptake behavior relied mainly on endocytosis, rather than physical adsorption or passive diffusion. In other words, cell uptake was energydependent. ${ }^{30}$ This cell-uptake ability indicated that the iRGD peptide can enhance the cell-uptake efficacy of $\mathrm{C}_{6}$-iRGD NPs in time-dependent, dosage-dependent, and energy-dependent manners, which is the main reason for the excellent efficacy of ISL-iRGD NPs in anti-breast cancer, rather than ISL-NPs or free ISL.

\section{In vivo antitumor efficacy of ISL-iRGD NPs}

Based on the strong sensitivity of the 4T1 cell line on ISLiRGD NPs, the classic 4T1-bearing nude-mouse model was selected to evaluate the in vivo anticancer efficacy of different formulations. Images of the tumors of the different groups are shown in Figure 5A. Further analysis comparing mean tumor volume between the ISL-iRGD NPs and the other three groups (free ISL, blank NPs, and control) showed significant difference $(P<0.05)$ was observed from the 14 th day (Figure 5B). For the end point, the mean tumor volume of ISL-iRGD NPs decreased to about $474 \mathrm{~mm}^{3}$ compared to the other three groups (Figure S3). In addition, the mean tumor-volume burden also confirmed a similar outcome with mean tumorvolume analysis. The mean tumor burden in ISL-iRGD NPs was lower then for free ISL, blank NPs, and the control group $(P<0.05)$, and slightly lower than the ISL NPs (Figure 5C). These results and the graphs indicate that ISL-iRGD NPs showed better anti-breast cancer efficacy than the other four groups (ISL NPs, free ISL, blank NPs, and control).

Furthermore, H\&E staining was used to record morphology changes among the different groups (Figure 5E). The quantity of mitotic-bodies in ISL-iRGD NPs and ISL NPs was higher than the other three groups, suggesting the viability of tumor cells was inhibited in ISL-iRGD NPs and ISL NPs in vivo. However, the blank NPs had similar morphology to the controls, which confirmed the low/absent anti-breast cancer effects of the nanomaterials.

For assessment of toxicity of the different formulas, body weight and H\&E staining of organs were used. Body weight varying with time was recorded for 20 days, and there was no significant difference among the five groups (Figure 5D), which showed these five formulae had no obvious side effects on the animals. On the other hand, H\&E staining of lungs, livers, and kidneys is shown in Figure S4. For lungs, all of the groups had lung metastasis detected, which met the basic characteristics of the 4T1-bearing mice model. For livers and kidneys, there were no remarkable morphological changes observed among the groups. All in all, this evidence confirmed the fact that our NPs had no obvious toxicity to the mice in this research.

As for dosage, $50 \mathrm{mg} / \mathrm{kg} /$ day was an effective dose for free ISL in nude mice in our preliminary studies. ${ }^{24,31-33}$ However, in this project the effective dose was decreased to $25 \mathrm{mg} / \mathrm{kg}$ every 2 days in the 4T1-bearing nude-mouse model. Also, the administration method was intraperitoneal rather than IV injection, which was because the volume of the NPs was too large to use IV injections. In sum, ISL-iRGD NPs had excellent ability for anti-breast cancer targeting in vivo with relative nontoxicity.

\section{Biodistribution characteristics of ISL-iRGD NPs}

In vitro targeting ability of ISL-iRGD NPs was evaluated by fluorescence microscopy and FCM in the foregoing sections, with satisfactory results, meeting our expectations. However, these findings focused only on manner of uptake at the cellular level. For passive uptake of NPs caused by the EPR effect, only in vivo research can be used. Moreover, in vivo biodistribution evaluation can show the targeting ability of ISL-iRGD NPs objectively and directly. Therefore, the in vivo biodistribution characteristics of ISL-iRGD analysis were assessed using the IVIS Spectrum in vivo imaging system.

Figure 6 shows the results of the biodistribution analysis of ISL-iRGD NPs. After IV injection of free Cy5, it was observed that the Cy5 accumulated mainly in the liver and intensity decreased at a higher speed than in the other two groups during the 24 hours. Moreover, little fluorescence was detected in tumors at 12 and 24 hours. However, in Cy5-iRGD NPs, fluorescence was detected at all four time points with relatively high intensity. Compared with $\mathrm{Cy} 5$ NPs, the detectable fluorescence area in tumors of Cy5-iRGD NPs was larger. The fluorescence signals of different organs or tissues in each group showed that the intensity of Cy5 in tumors followed an ascending order: Free Cy5, Cy5 NPs, and Cy5-iRGD NPs $(P<0.05)$. In addition, Cy5 was mainly accumulated in the liver with stronger intensity compared to the other two groups $(P<0.05)$. All in all, based on biodistribution analysis in vivo, Cy5-iRGD NPs showed better tumor-targeting ability than free Cy5 and Cy5 NPs, perhaps due to the tumor-homing iRGD peptide and EPR effect. 
A
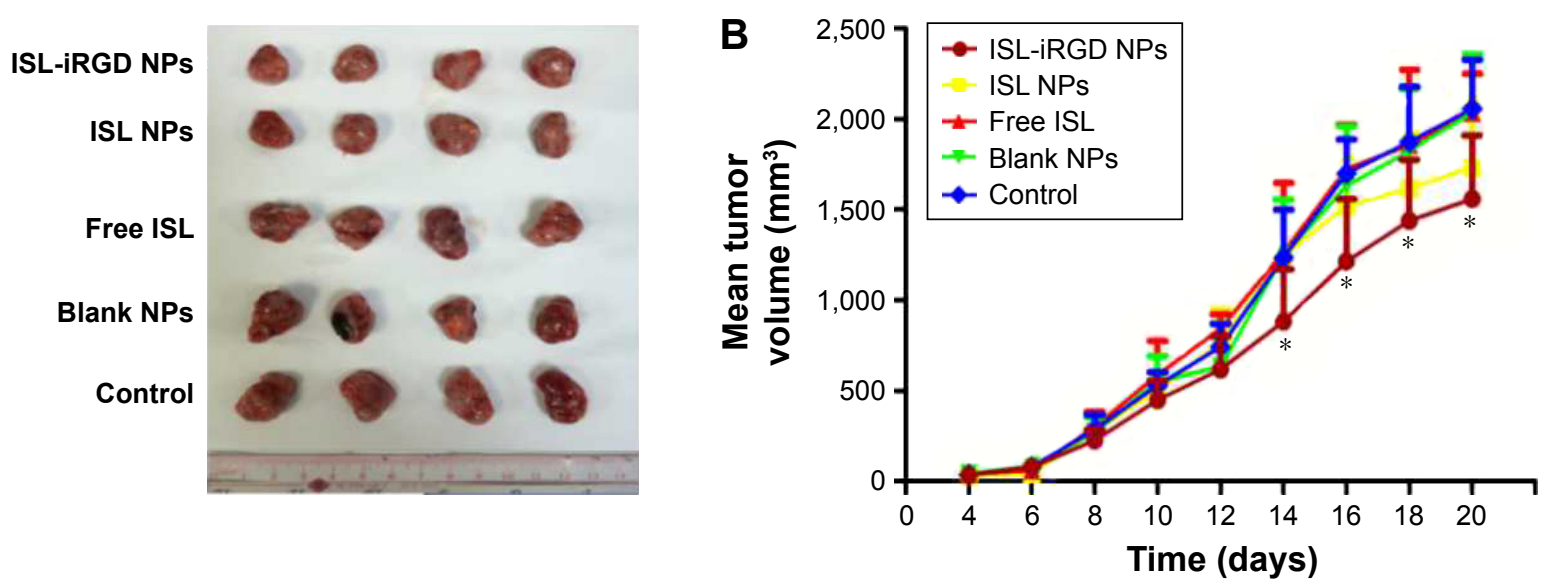

C

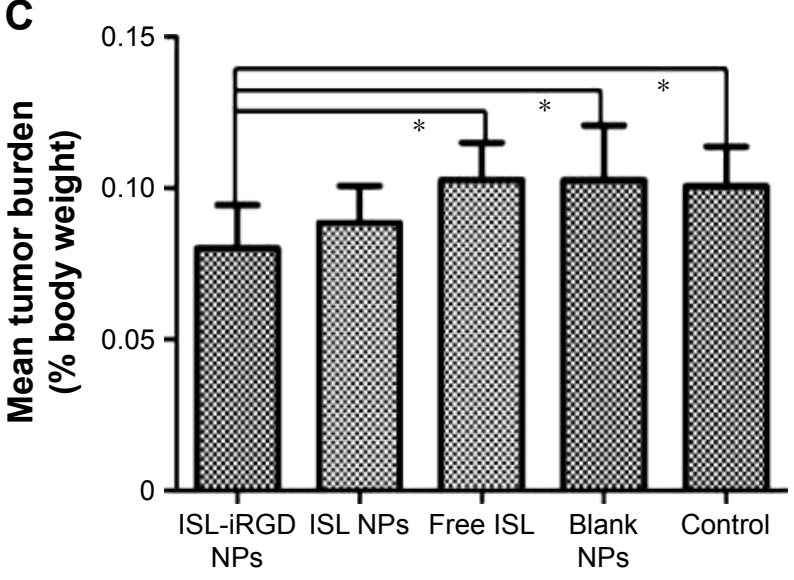

E

ISL-iRGD NPs

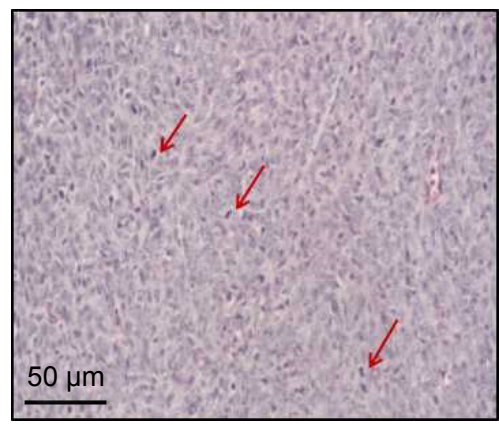

ISL NPs

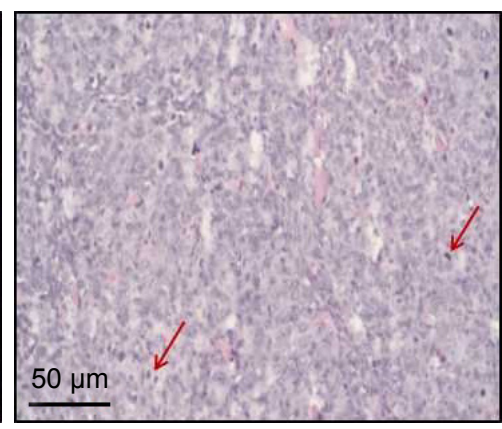

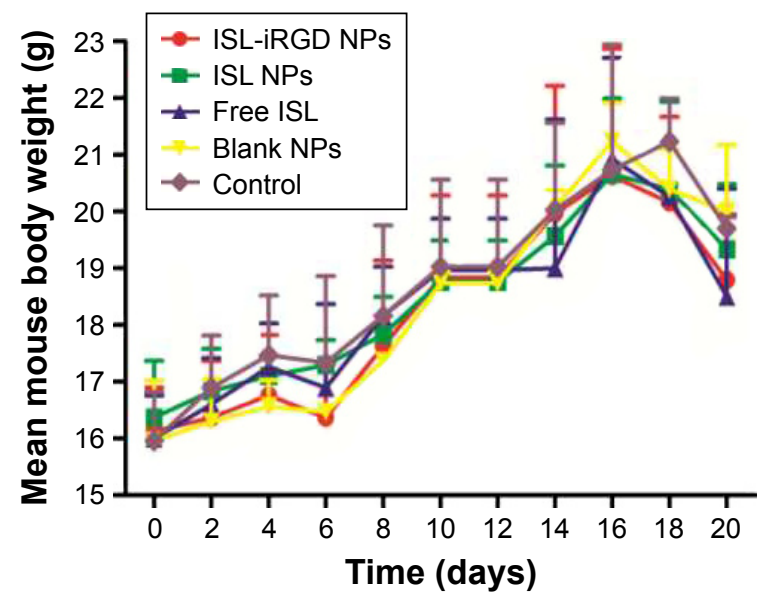

Free ISL

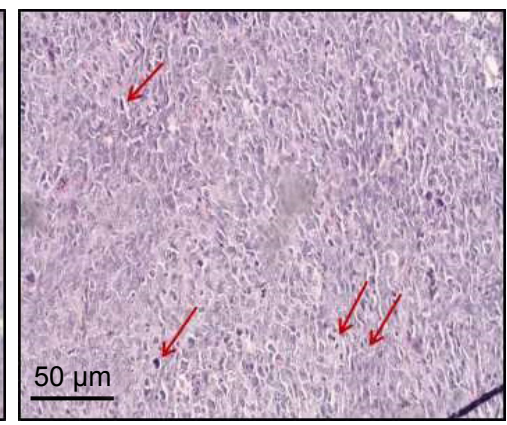

Control
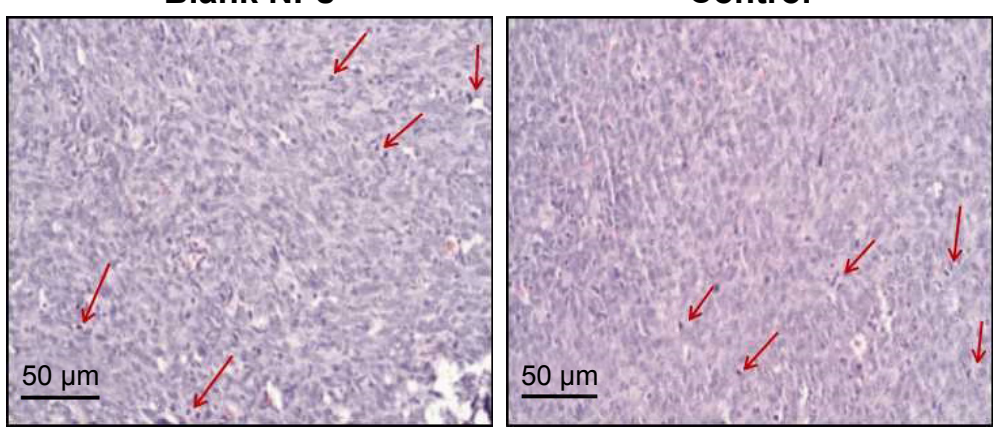

Figure 5 In vivo antitumor efficacy of ISL-iRGD NPs, ISL NPs, free ISL, blank NPs, and saline in the tumor-bearing mouse model.

Notes: (A) Graph of the tumor after intraperitoneal injection of different ISL formulations ( $25 \mathrm{mg} / \mathrm{kg})$; (B) profile of mean tumor volume varying with time among different groups ( $* P<0.05$ vs free ISL, blank NPs, and saline); (C) mean tumor burden among different ISL formulations $(* P<0.05)$; (D) profile of the mean mouse body weight varying with time among different groups; (E) H\&E staining on tumor $(\times 20$ objective). The red arrows indicate the mitotic-bodies of the cancer cells.

Abbreviations: ISL, isoliquiritigenin; NPs, nanoparticles; H\&E, hematoxylin and eosin. 

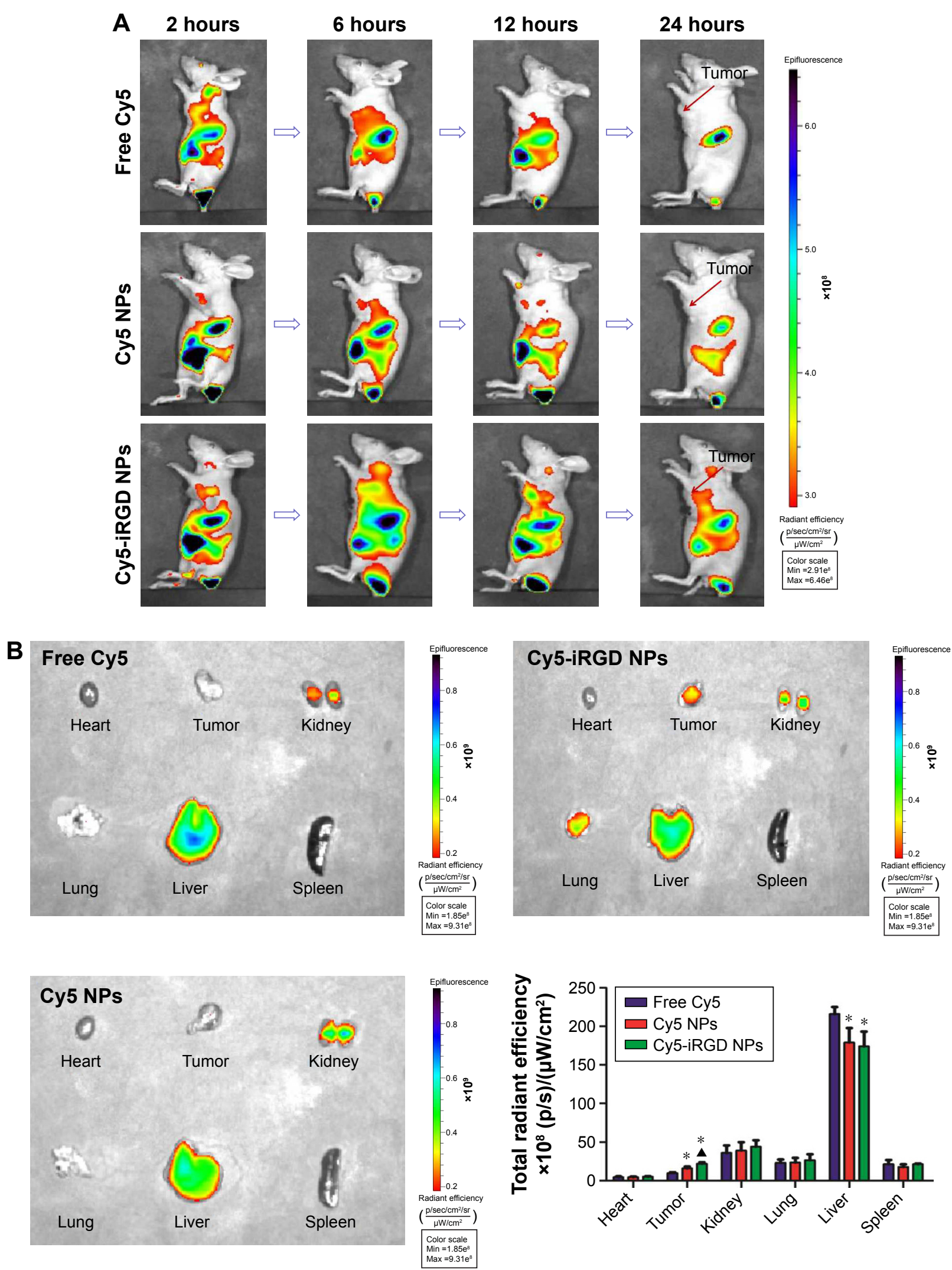

Figure 6 Biodistribution analysis of free Cy5, Cy5 NPs, and Cy5-iRGD NPs in the tumor-bearing mouse model.

Notes: Mice were injected intravenously via the tail vein with free Cy5, Cy5 NPs, and Cy5-iRGD NPs ( $35 \mu g / k g$ mice). (A) Fluorescence images of mice treated with different Cy5 formulations in vivo at different time points (2, 6, 12, and 24 hours). (B) Fluorescence images of mice organs treated with different Cy5 formulations for 24 hours in vivo and fluorescent-signal analysis of different organs/tissues in each group. $* P<0.05$ compared with free Cy5; ${ }^{\Delta} P<0.05$ compared with Cy5 NPs.

Abbreviations: ISL, isoliquiritigenin; NPs, nanoparticles. 


\section{Conclusion}

In this study, ISL-iRGD NPs were prepared successfully. The size of the NPs was about $130 \mathrm{~nm}$, which is the appropriate size for the EPR effect in cancer therapy. ${ }^{28}$ The $\zeta$-potential of ISL-iRGD NPs was about $-34 \mathrm{mV}$, which indicated a relatively stable system. ${ }^{29}$ ISL-iRGD NP structure was also confirmed by four different methods, which demonstrated that the ISL was successfully encapsulated in the core of the NPs with high stability. In addition, they also had sufficient entrapment efficiency and loading efficiency. Compared with ISL NPs and free NPs, ISL-iRGD NPs had better efficacy in anti-breast cancer targeting and stronger targeting ability. In the pharmacodynamic study in vitro, ISL-iRGD NPs showed stronger inhibition effects and induced apoptosis effects. In the in vivo research, ISL-iRGD NPs showed stronger effects in inhibiting tumor growth and viability of tumor cells. The toxicity of the nanomaterials was also evaluated. The in vivo study outcome showed the nanomaterials had no significant damaging effects on normal tissue. The targeting ability of ISL-iRGD NPs was detected in vivo and in vitro. The data showed that ISL-iRGD NPs had better ability in increasing cell-uptake efficiency in a time-independent, dose-independent, and energy-independent manner. The biodistribution test also indicated that ISL-iRGD NPs were easier to target and accumulate in breast tumors. All in all, this new delivery system for encapsulating ISL was effective and can promote the development of ISL in breast cancer therapy in clinical practice.

The global burden of cancer has been increasing in the past few decades, raising the prospect of significant investment in health systems and thus posing a real medical problem. According to GloboCan data in 2012, breast cancer is the commonest cancer in women worldwide. Due to the serious toxicity of first-line synthetic agents, the high incidence of breast cancer and its correlation with cancerrelated deaths worldwide have prompted more attention on discovering novel and effective alternatives with fewer side effects. Natural products, such as dietary phytochemicals, tea polyphenols, and resveratrol, are well recognized as effective agents in several human ailments, including cancer. A natural flavonoid, ISL has previously been demonstrated to exhibit antioxidant, anti-inflammatory, and tumor-suppression effects by inducing apoptosis, autophagy, and cell-growth inhibition. Nevertheless, the high lipophilicity and insufficient anticancer efficacy in comparison with synthetic agents like doxorubicin greatly limit its clinical efficacy. Recently, nanoscale carriers have played a significant role in drug delivery. Various types of nanovehicles, such as liposomes, micelles, and nanocapsules, can encapsulate poorly soluble drugs to improve solubility. More importantly, anticancer drugs loaded in NPs can easily permeate into the leaky vasculature of tumor-tissue and control the drug-release behavior at the tumor site, which has been well described to enhance anticancer efficiency.

Lipid-related NPs, with liposomes as the classic type, and polymer-manufactured NPs, such as polymeric micelles and dendrimers, are the two dominant classes of nanoscale drug vehicles, with the increasing backing of approved drug products, clinical trials, and research experiments. Although many advantages of these nanocarriers have been demonstrated on occasion to improve solubility, body circulation, and selective tumor distribution, unfortunately both classes suffer limitations in terms of their physicochemical and biological properties. Specifically, most liposomes exhibit superior biocompatibility, biodegradability, and nonimmunogenic response, because natural lipids are analogs of biological membranes. However, liposomes display poor storage stability, and are easily cleared by the reticuloendothelial system. On the other hand, polymeric NPs have advantages in particle size, stability, and drug loading. Therefore, herein we employed lipid-polymer hybrid NPs constituted of PLGA, lecithin, and PEG to combine the advantages of lipid NPs and polymeric NPs. Moreover, iRGD peptides were grafted to DSPE-PEG ${ }_{2,000}$-Mal to obtain tumor-targeting by the conjugation of the thiol group of iRGD to the Mal group of PEG. ${ }^{30}$ Our research confirmed significant targeting ability and anti-breast cancer effects both in vivo and in vitro. How to synthesize such materials using simpler and cost-effective methods would be the key question, which requires addressing.

\section{Acknowledgments}

This study was supported by Seed Funding in HKU (201511160021 and 201409160015) and Chinese National Natural Science Foundation (81573663). The project was also funded by the Talent Training Program, 2011 Cooperation Program of Chengdu University of Traditional Chinese Medicine. The abstract of this paper was presented at the 15th World Congress on Cancer Therapy, Biomarkers, and Clinical Research, December 5-7, 2016 in Philadelphia, PA.

\section{Disclosure}

The authors report no conflicts of interest in this work. 


\section{References}

1. DeSantis CE, Fedewa SA, Sauer AG, Kramer JL, Smith RA, Jemal A. Breast cancer statistics, 2015: convergence of incidence rates between black and white women. CA Cancer J Clin. 2016;66(1):31-42.

2. Ferlay J, Soerjomataram I, Dikshit R, et al. Cancer incidence and mortality worldwide: sources, methods and major patterns in GLOBOCAN 2012. Int J Cancer. 2015;136(5):E359-E386.

3. Cardoso F, Harbeck N, Fallowfield L, Kyriakides S, Senkus E. Locally recurrent or metastatic breast cancer: ESMO clinical practice guidelines for diagnosis, treatment and follow-up. Ann Oncol. 2012;23 Suppl 7: vii11-vii19.

4. Fridman WH, Pagès F, Sautès-Fridman C, Galon J. The immune contexture in human tumours: impact on clinical outcome. Nat Rev Cancer. 2012;12(4):298-306

5. Goldfarb Y, Sorski L, Benish M, Levi B, Melamed R, Ben-Eliyahu S. Improving postoperative immune status and resistance to cancer metastasis: a combined perioperative approach of immunostimulation and prevention of excessive surgical stress responses. Ann Surg. 2011; 253(4):798-810

6. Croker AK, Allan AL. Inhibition of aldehyde dehydrogenase (ALDH) activity reduces chemotherapy and radiation resistance of stem-like ALDHhiCD44+ human breast cancer cells. Breast Cancer Res Treat. 2012;133(1):75-87.

7. Vinod BS, Maliekal TT, Anto RJ. Phytochemicals as chemosensitizers: from molecular mechanism to clinical significance. Antioxid Redox Signal. 2013;18(11):1307-1348.

8. Wang Z, Wang N, Han S, et al. Dietary compound isoliquiritigenin inhibits breast cancer neoangiogenesis via VEGF/VEGFR-2 signaling pathway. Plos One. 2013;8(7):e68566.

9. Wang N, Wang Z, Wang Y, et al. Dietary compound isoliquiritigenin prevents mammary carcinogenesis by inhibiting breast cancer stem cells through WIF1 demethylation. Oncotarget. 2015;6(12):9854-9876.

10. Wang N, Wang Z, Peng C, et al. Dietary compound isoliquiritigenin targets GRP78 to chemosensitize breast cancer stem cells via $\beta$-catenin/ ABCG2 signaling. Carcinogenesis. 2014;35(11):2544-2554.

11. Wang Z, Wang N, Liu P, et al. MicroRNA-25 regulates chemoresistanceassociated autophagy in breast cancer cells, a process modulated by the natural autophagy inducer isoliquiritigenin. Oncotarget. 2014; 5(16):7013-7026.

12. Li Y, Zhao H, Wang $\mathrm{Y}$, et al. Isoliquiritigenin induces growth inhibition and apoptosis through downregulating arachidonic acid metabolic network and the deactivation of PI3K/Akt in human breast cancer. Toxicol Appl Pharmacol. 2013;272(1):37-48.

13. Lorusso V, Marech I. Novel plant-derived target drugs: a step forward from licorice? Expert Opin Ther Targets. 2013;17(4):333-335.

14. Jang DS, Park EJ, Hawthorne ME, et al. Potential cancer chemopreventive constituents of the seeds of Dipteryx odorata (tonka bean). J Nat Prod. 2003;66(5):583-587.

15. Cuendet M, Guo J, Luo Y, et al. Cancer chemopreventive activity and metabolism of isoliquiritigenin, a compound found in licorice. Cancer Prev Res (Phila). 2010;3(2):221-232.

16. Gao H, Yang Z, Zhang S, et al. Ligand modified nanoparticles increases cell uptake, alters endocytosis and elevates glioma distribution and internalization. Sci Rep. 2013;3:2534.
17. Hadinoto K, Sundaresan A, Cheow WS. Lipid-polymer hybrid nanoparticles as a new generation therapeutic delivery platform: a review. Eur J Pharm Biopharm. 2013;85(3 Pt A):427-443.

18. Mandal B, Bhattacharjee H, Mittal N, et al. Core-shell-type lipidpolymer hybrid nanoparticles as a drug delivery platform. Nanomedicine. 2013;9(4):474-491.

19. Byrne JD, Betancourt T, Brannon-Peppas L. Active targeting schemes for nanoparticle systems in cancer therapeutics. Adv Drug Deliv Rev. 2008;60(15):1615-1626.

20. Ruoslahti E. Specialization of tumour vasculature. Nat Rev Cancer. 2002;2(2):83-90.

21. Murphy EA, Majeti BK, Barnes LA, et al. Nanoparticle-mediated drug delivery to tumor vasculature suppresses metastasis. Proc Natl Acad Sci US A. 2008;105(27):9343-9348.

22. Alberici L, Roth L, Sugahara KN, et al. De novo design of a tumorpenetrating peptide. Cancer Res. 2013;73(2):804-812.

23. Sugahara KN, Teesalu T, Karmali PP, et al. Coadministration of a tumor-penetrating peptide enhances the efficacy of cancer drugs. Science. 2010;328(5981):1031-1035.

24. Zhang XY, Qiao H, Ni JM, Shi YB, Qiang Y. Preparation of isoliquiritigenin-loaded nanostructured lipid carrier and the in vivo evaluation in tumor-bearing mice. Eur J Pharm Sci. 2013;49(3): 411-422.

25. Han B, Zhang MM, Tang T, et al. The long-term fate and toxicity of PEG-modified single-walled carbon nanotube isoliquiritigenin delivery vehicles in rats. J Nanomater. 2014;2014:257391.

26. Su XW, Wang ZH, Li LL, et al. Lipid-polymer nanoparticles encapsulating doxorubicin and 2 -deoxy-5-azacytidine enhance the sensitivity of cancer cells to chemical therapeutics. Mol Pharm. 2013;10(5): 1901-1909.

27. Conner SD, Schmid SL. Regulated portals of entry into the cell. Nature. 2003;422(6927):37-44.

28. Zhang J, Li Y, Gao W. Correction to: Andrographolide-loaded PLGAPEG-PLGA micelles to improve its bioavailability and anticancer efficacy. Expert Opin Drug Deliv. 2015;12(4):689.

29. Wang S, Chen R, Morott J, Repka MA, Wang Y, Chen M. mPEG-b-PCL/ TPGS mixed micelles for delivery of resveratrol in overcoming resistant breast cancer. Expert Opin Drug Deliv. 2015;12(3):361-373.

30. Kam NW, Liu ZA, Dai HJ. Carbon nanotubes as intracellular transporters for proteins and DNA: an investigation of the uptake mechanism and pathway. Angew Chem Int Ed Engl. 2006;45(4):577-581.

31. Wang ZY, Wang N, Liu PX, et al. MicroRNA-25 regulates chemoresistance-associated autophagy in breast cancer cells, a process modulated by the natural autophagy inducer isoliquiritigenin. Oncotarget. 2014;5(16):7013-7026.

32. Peer D, Karp JM, Hong S, Farokhzad OC, Margalit R, Langer R. Nanocarriers as an emerging platform for cancer therapy. Nat Nanotechnol. 2007;2(12):751-760.

33. Arias JL, López-Viota M, Clares B, Ruiz MA. Stability of fenbendazole suspensions for veterinary use: correlation between zeta potential and sedimentation. Eur J Pharm Sci. 2008;34(4-5):257-262. 


\section{Supplementary materials}
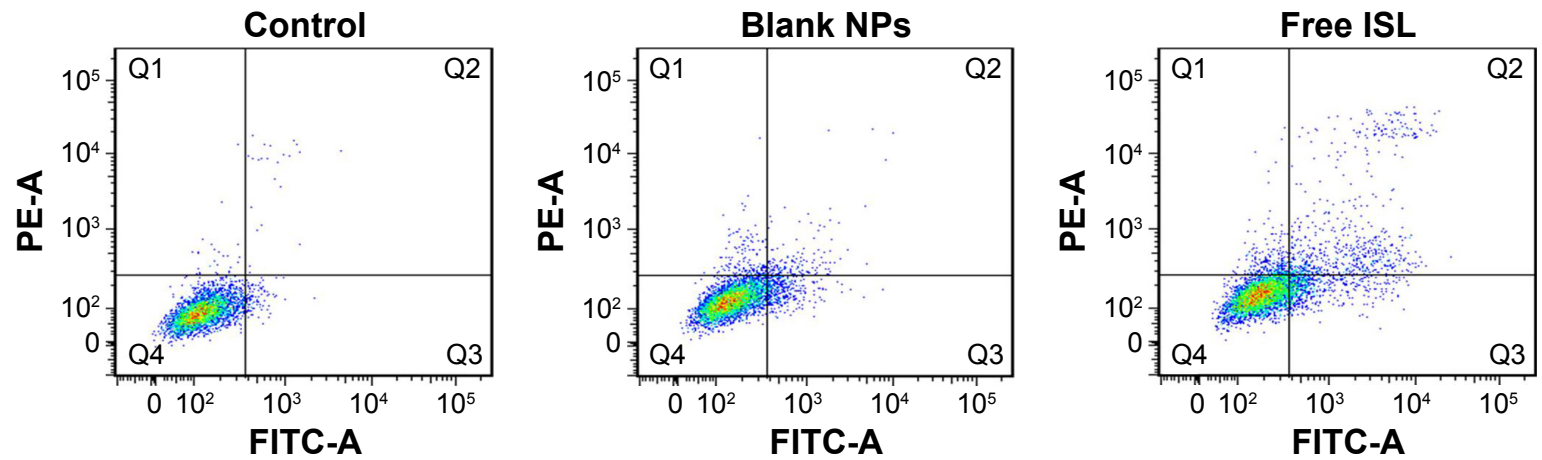

ISL NPs
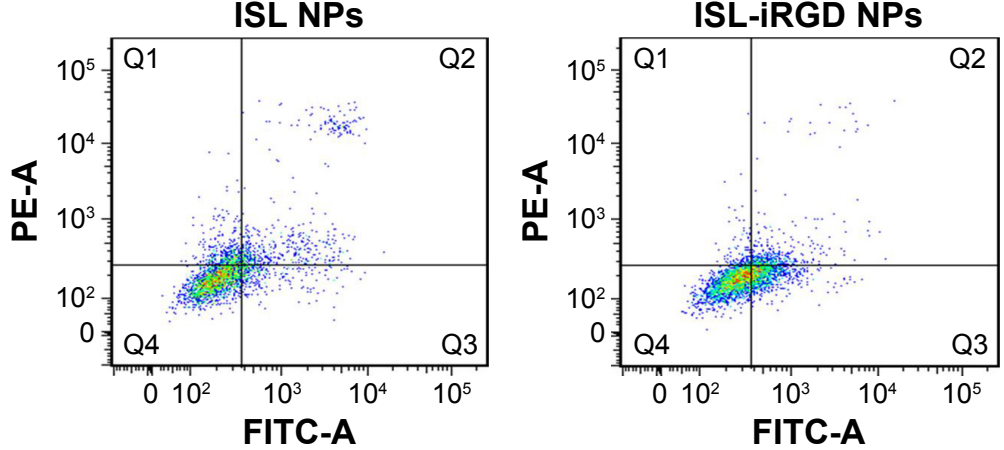

Figure SI Flow cytometry of different ISL formulations for apoptosis analysis in MDA-MB23 I cell lines.

Notes: Top left (QI), necrotic cells (annexin $\mathrm{V}_{-} \mathrm{FITC}^{-} \mathrm{PI}^{+}$); top right (Q2), late apoptotic cells (annexin $\mathrm{V}-\mathrm{FITC}^{+} \mathrm{PI}^{+}$); bottom left (Q3), live cells (annexin $\mathrm{V}-\mathrm{FITC}^{-} \mathrm{PI}^{-}$); bottom right (Q4), early apoptotic cells (annexin $\mathrm{V}-\mathrm{FITC}^{+} \mathrm{PI}^{-}$).

Abbreviations: ISL, isoliquiritigenin; PI, propidium iodide; NPs, nanoparticles.

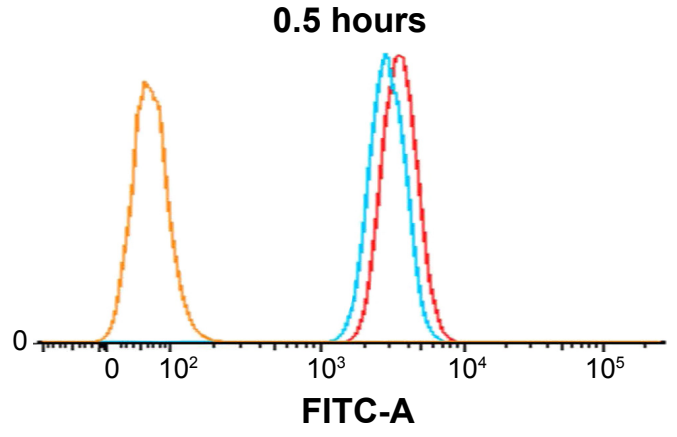

2 hours

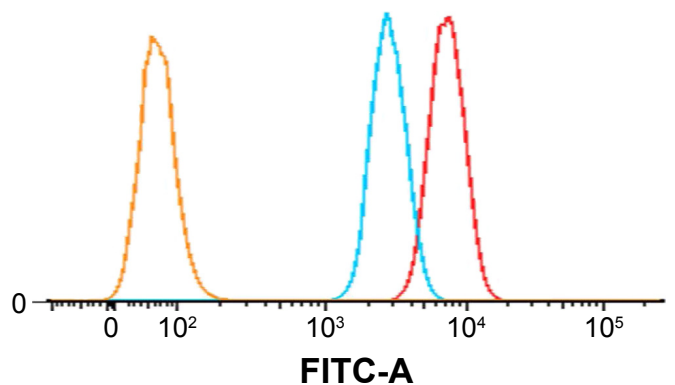

1 hour

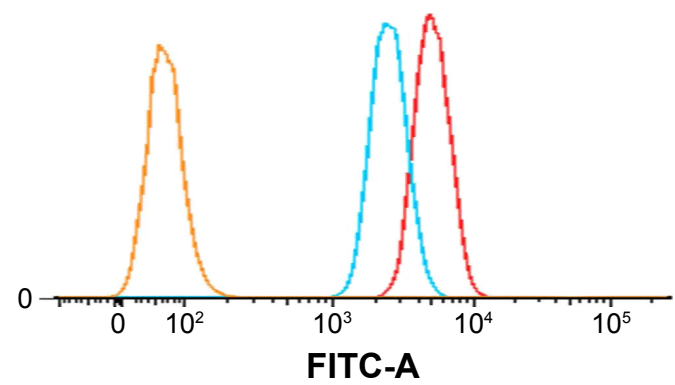

4 hours

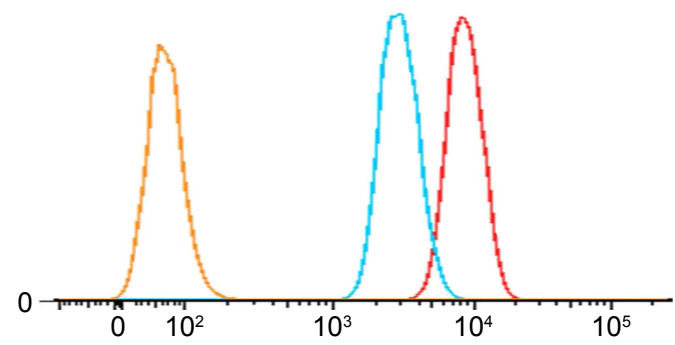

FITC-A

- Control $-4^{\circ} \mathrm{C}-37^{\circ} \mathrm{C}$

Figure S2 Flow-cytometry profile of MDA-MB23I cell line incubated with $\mathrm{C}_{6}$-iRGD NPs at different incubation time points $\left(0.5, \mathrm{I}, 2\right.$, and 4 hours) at $4^{\circ} \mathrm{C}$ and $37^{\circ} \mathrm{C}$. Abbreviation: NPs, nanoparticles. 


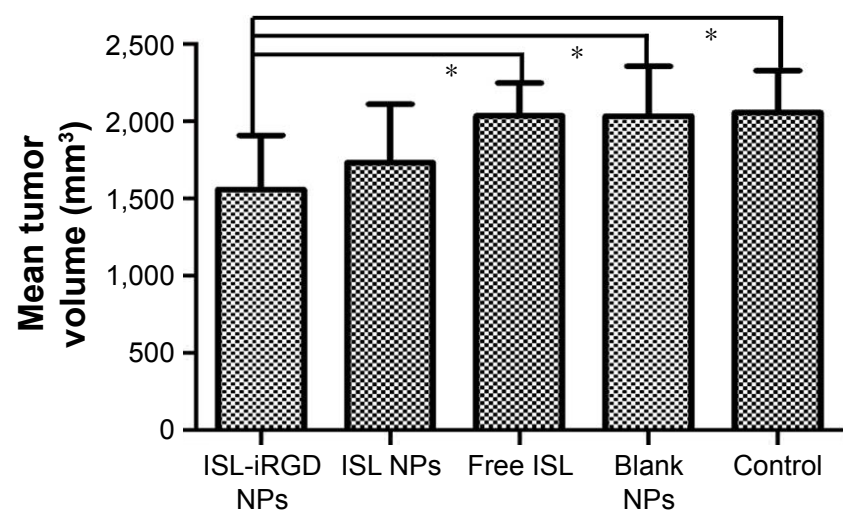

Figure S3 Mean tumor volume differences among different groups $(* P<0.05)$. Abbreviations: ISL, isoliquiritigenin; NPs, nanoparticles.
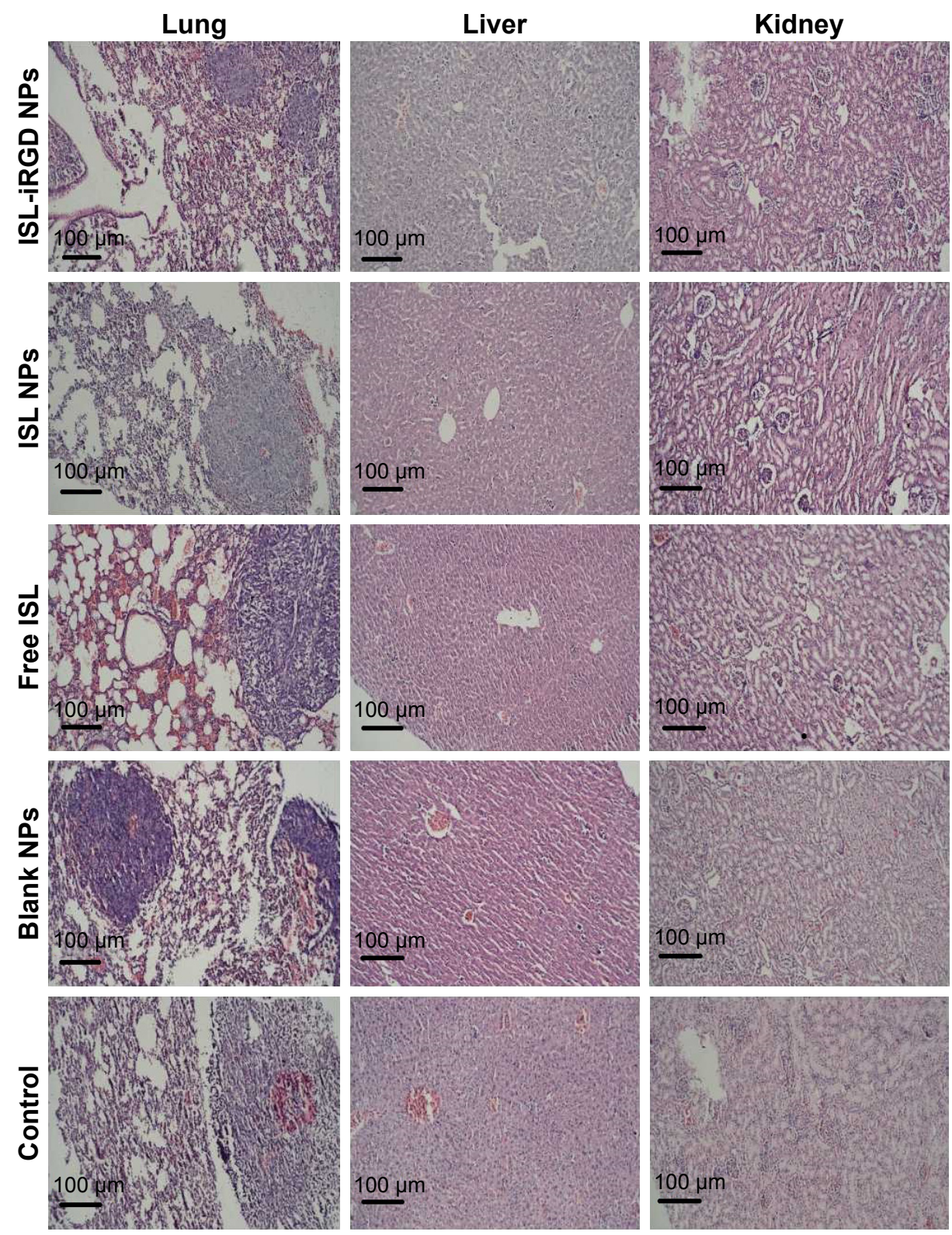

Figure S4 Hematoxylin and eosin staining on lung, liver, and kidney (10× objective). Abbreviations: ISL, isoliquiritigenin; NPs, nanoparticles. 


\section{Publish your work in this journal}

The International Journal of Nanomedicine is an international, peerreviewed journal focusing on the application of nanotechnology in diagnostics, therapeutics, and drug delivery systems throughout the biomedical field. This journal is indexed on PubMed Central, MedLine, CAS, SciSearch $\AA$, Current Contents $\AA /$ Clinical Medicine,
Journal Citation Reports/Science Edition, EMBase, Scopus and the Elsevier Bibliographic databases. The manuscript management system is completely online and includes a very quick and fair peer-review system, which is all easy to use. Visit http://www.dovepress.com/ testimonials.php to read real quotes from published authors.

Submit your manuscript here: http://www.dovepress.com/international-journal-of-nanomedicine-journal 